\title{
The hidden flat like universe
}

\section{Starobinsky-like inflation induced by $f(T)$ gravity}

\author{
W. El Hanafy ${ }^{1,3, a}$, G. G. L. Nashed ${ }^{1,2,3, b}$ \\ ${ }^{1}$ Centre for Theoretical Physics, The British University in Egypt, P.O. Box 43, El Sherouk 11837, Egypt \\ ${ }^{2}$ Mathematics Department, Faculty of Science, Ain Shams University, Cairo, Egypt \\ ${ }^{3}$ Egyptian Relativity Group, Giza, Egypt
}

Received: 10 April 2015 / Accepted: 2 June 2015 / Published online: 24 June 2015

(C) The Author(s) 2015. This article is published with open access at Springerlink.com

\begin{abstract}
We study a single-fluid component in a flat like universe (FLU) governed by $f(T)$ gravity theories, where $T$ is the teleparallel torsion scalar. The FLU model, regardless of the value of the spatial curvature $k$, identifies a special class of $f(T)$ gravity theories. Remarkably, FLU $f(T)$ gravity does not reduce to teleparallel gravity theory. In large Hubble spacetime the theory is consistent with the inflationary universe scenario and respects the conservation principle. The equation of state evolves similarly in all models $k=0, \pm 1$. We study the case when the torsion tensor consists of a scalar field, which enables to derive a quintessence potential from the obtained $f(T)$ gravity theory. The potential produces Starobinsky-like model naturally without using a conformal transformation, with higher orders continuously interpolate between Starobinsky and quadratic inflation models. The slow-roll analysis shows double solutions, so that for a single value of the scalar tilt (spectral index) $n_{s}$ the theory can predict double tensor-to-scalar ratios $r$ of $E$-mode and $B$-mode polarizations.
\end{abstract}

\section{Introduction}

The general relativity (GR) theory explained the gravity as spacetime curvature. This description of gravitation has succeeded to confront astrophysical observations for a long time. It has predicted perfectly the perihelion shift of mercury, time delay in the solar system. Even in the strong field regimes such as binary pulsars it has amazingly predicted their orbital decay due to gravitational radiation by the system. While it fails to predict the accelerating cosmic expansion which is evidenced by the astronomical observations of high-redshift Type Ia supernovae [1]. The teleparallel equivalent of general

\footnotetext{
a e-mail: waleed.elhanafy@bue.edu.eg

b e-mail: nashed@bue.edu.eg
}

relativity (TEGR) theory has provided an alternative description of Einstein's gravity. The theory constructed from vierbein (tetrad) fields ${ }^{1}\left\{h^{a}{ }_{\mu}\right\}$ instead of metric tensor fields $g_{\mu \nu}$. The metric space, however, can be constructed from the vierbein fields, so the Levi-Civita symmetric connection $\stackrel{\circ}{\Gamma}^{\alpha} \mu \nu$. It is, also, possible to construct Weitzenböck nonsymmetric connection $\Gamma_{\mu \nu}^{\alpha}$. The first connection has a non-vanishing curvature tensor $R_{\alpha \beta \mu \nu}^{(\stackrel{\circ}{\Gamma})} \neq 0$ but a vanishing torsion tensor $T_{\alpha \mu \nu}^{(\stackrel{\circ}{\Gamma})}=0$, while the later is characterized by a vanishing curvature tensor $R_{\alpha \beta \mu \nu}^{(\Gamma)}=0$ but a non-vanishing torsion tensor $T_{\alpha \mu \nu}^{(\Gamma)} \neq 0$. The combined picture could be encoded in the contracted Bianchi identity

$R^{(\stackrel{\circ}{\Gamma})} \equiv-T^{(\Gamma)}-2 \nabla_{\alpha}^{(\stackrel{\circ}{\Gamma})} T^{v \alpha}{ }_{\nu}$

where $R^{(\stackrel{\circ}{\Gamma})}$ is the usual Ricci scalar, the scalar invariant $T^{(\Gamma)}$ is called the teleparallel torsion scalar (it will be briefly investigated in Sect. 2 and the covariant derivative $\nabla^{(\stackrel{\circ}{\Gamma})}$ is with respect to (w.r.t.) the Levi-Civita connection. The variation of the left hand side w.r.t. the metric tensor provides the GR field equations. Since the last term in the identity is a total derivative, it does not contribute to the field equations, and the variation of the right hand side w.r.t. the vierbein fields provides a set of field equations equivalent to the GR that is called TEGR. Although the two theories are quantitatively equivalent at their level of the field equations, they are qualitatively different at the level of their actions! Indeed, the total derivative term is scalar invariant under a diffeomorphism but not invariant under a local Lorentz transformation (LLT). On the other hand, the Ricci scalar in the Einstein-Hilbert action leads to a theory invariant under a diffeomorphism as well

\footnotetext{
1 The Greek letters $\alpha, \beta, \ldots$ denote the spacetime indices and the Latin ones $a, b, \ldots$ denote Lorentz indices. Both run from 0 to 3 .
} 
as LLT. Consequently, the teleparallel torsion scalar $T$ is not invariant under LLT [2]. The absence of local Lorentz symmetry in the TEGR action will not be reflected in the field equations, so it does not seem worth to worry about it. However, the presence of the total derivative term is crucial when we consider the $f(T)$ extension of TEGR $[3,4]$.

Teleparallel geometry has received attention in the last decade. However, this geometry has been used very much earlier, in the 1920s, to unify gravity and electromagnetism by Einstein [5]. After this trial the geometry has been developed [6-8]. Later, successful extensions to Einstein's work allowed a class of theories with a quadratic torsion in Lagrangian density [9-11]. Other trials to obtain a gauge field theory of gravity using the teleparallel geometry have shown to be of great interest [12-16]. Recent developments attempted a global approach by using arbitrary moving frames instead of the local expressions in the natural basis $[17,18]$. Also, it is worth to mention other developments in teleparallel geometry: by imposing Finslerian properties to this geometrical structure [19-21]. Actually, this geometry provides an alternative tool for deeper studies of gravity.

An interesting variant of the TEGR is the Born-Infeldmodified teleparallel gravity. Within this framework the early cosmic acceleration (inflation) could be accounted for with no need of an inflaton field [22,23]. Another remarkable variant of generalizations of TEGR are the $f(T)$ theories similar to the $f(R)$ extensions of the Einstein-Hilbert action. Within this new class of modified gravity theories a particular form has been proposed to explain the late-time cosmic speeding up without dark energy (DE) [24-27]. As is well known $f(R)$ theories are conformally equivalent to the EinsteinHilbert action plus a scalar field. In contrast, the $f(T)$ theories cannot be conformally equivalent to TEGR plus a scalar field [28]. The short period of these pioneering studies has been followed by a large number of papers exploring different aspects of the $f(T)$ gravity in astrophysics [29-40] and in cosmology [41-50]. Some applications show interesting results, e.g. avoiding the big bang singularity by presenting a bouncing solution [51,52]. Also, a graceful exit inflationary model within the $f(T)$ cosmology has been argued for in [53]. A recent promising variant is the modified teleparallel equivalent of Gauss-Bonnet gravity and its applications [54-56].

Spatially flat universe (SFU), i.e. $k=0$, is used widely in the literature seeking for consistency with cosmic observations. However, recent observations by the Planck satellite suggest also an open universe as a reliable model [57]. On the other hand, the smallness of the curvature density parameter cannot be covered by the SFU assumption. In the present study, instead of restricting ourselves to SFU, we impose FLU assumptions onto the modified $f(T)$ Friedmann equations. This model identifies a hidden class of $f(T)$ gravity theories that cannot be covered by assuming a SFU. The rest of this paper is structured as follows: in Sect. 2, we briefly review teleparallel geometry and the $f(T)$ gravity theories. In Sect. 3, we use the modified version of the Friedmann equations according to $f(T)$ gravity to describe a FLU. In Sect. 4 , we discuss the physical and cosmological consequences of the obtained results. In Sect.5, we assume the case when the torsion tensor consists of a scalar field. This enables us to construct an exact inflation model powered by a quintessence-like field sensitive to the spacetime symmetry. In addition, it enables us to induce a generalized Starobinsky potential from the obtained $f(T)$ gravity theory. In Sect. 6, we study possible solutions of the slow-roll parameters of this theory. We discuss testable predictions of the theory according to the recently released Planck and BICEP2 data [57-59]. The work is summarized in Sect. 7.

\section{Extended teleparallel gravity}

In what follows, we give the structure of Weitzenböck 4-space. It is described as a pair $\left(M, h_{a}\right)$, where $M$ is a 4-dimensional smooth manifold and $h_{a}(a=0, \ldots, 3)$ are 4-linearly independent vector (vierbein) fields defined globally on $M$. Consequently, the $|h|:=\operatorname{det}\left(h_{a}^{\mu}\right)$ is nonzero. The vierbein fields and their dual coframes are orthonormal, i.e. $h_{a}{ }^{\mu} h_{v}^{a}=\delta_{v}^{\mu}$ and $h_{a}{ }^{\mu} h_{\mu}^{b}=\delta_{a}^{b}$, where $\delta$ is the Kronecker tensor. The metric space can be constructed from the vierbein fields $g_{\mu \nu}:=\eta_{a b} h^{a}{ }_{\mu} h^{b}{ }_{\nu}$ where $\eta_{a b}=\operatorname{diag}(1,-1,-1,-1)$ is the Minkowski metric for the tangent space, so the Riemannian geometry is recovered. It is also possible to construct the Weitzenböck nonsymmetric connection,

$\Gamma_{\mu \nu}^{\alpha}:=h_{\mu}^{a} \partial_{\nu} h_{a}^{\alpha}=-h_{a}{ }^{\alpha} \partial_{\nu} h_{\mu}^{a}$,

where $\partial_{v}=\frac{\partial}{\partial x^{v}}$. The Weitzenböck space is characterized by the vanishing of the vierbein's covariant derivative, i.e.

$\nabla_{\nu}^{(\Gamma)} h_{\mu}^{a}:=\partial_{\nu} h_{a}{ }^{\mu}+\Gamma_{\lambda \nu}^{\mu} h_{a}{ }^{\lambda} \equiv 0$,

where the covariant derivative $\nabla^{(\Gamma)}$ is w.r.t. the Weitzenböck connection. So this property identifies the auto parallelism or absolute parallelism condition. As a matter of fact, the $\nabla^{(\Gamma)}$ operator is not covariant under local Lorentz transformations (LLT) $S O(3,1)$ allowing all LLT invariant geometrical quantities to rotate freely in every point of the space [60]. In this sense, the symmetric metric (10 degrees of freedom) cannot predict exactly one set of vierbein fields; then the extra six degrees of freedom of the 16 vierbein fields need to be fixed in order to identify exactly one physical frame. The frames $h_{a}$ are called the parallelization vector fields. It can be shown that the teleparallelism condition (2) implies the metricity condition $\nabla_{\sigma}^{(\stackrel{\circ}{\Gamma})} g_{\mu \nu} \equiv 0$. The Weitzenböck connection (1) is curvature free, while it has a torsion tensor. The torsion $T$ and the contortion $K$ tensor fields of type $(1,2)$ in spacetime 
coordinates are

$T_{\mu \nu}^{\alpha}:=\Gamma_{\nu \mu}^{\alpha}-\Gamma_{\mu \nu}^{\alpha}=h_{a}^{\alpha}\left(\partial_{\mu} h^{a}{ }_{\nu}-\partial_{\nu} h_{\mu}^{a}\right)$,

$K_{\alpha}^{\mu \nu}:=-\frac{1}{2}\left(T_{\alpha}^{\mu \nu}-T_{\alpha}^{v \mu}-T_{\alpha}^{\mu \nu}\right)$.

In the teleparallel space one may define three Weitzenböck invariants: $I_{1}=T^{\alpha \mu \nu} T_{\alpha \mu \nu}, I_{2}=T^{\alpha \mu \nu} T_{\mu \alpha \nu}$, and $I_{3}=T^{\alpha} T_{\alpha}$, where $T^{\alpha}=T_{\nu}{ }^{\alpha \nu}$. We next define the invariant $T=A I_{1}+B I_{2}+C I_{3}$, where $A, B$, and $C$ are arbitrary constants [60]. For the values: $A=1 / 4, B=1 / 2$, and $C=-1$ the invariant $T$ is just the Ricci scalar $R^{(\stackrel{\circ}{\Gamma})}$, up to a total derivative term as mentioned in Sect. 1; then a teleparallel version of gravity equivalent to GR can be achieved. The teleparallel torsion scalar is given in the compact form

$T:=T_{\mu \nu}^{\alpha} S_{\alpha}^{\mu \nu}$,

where the superpotential tensor $S_{\alpha}^{\mu \nu}$ is defined as

$S_{\alpha}^{\mu v}:=\frac{1}{2}\left(K_{\alpha}^{\mu \nu}+\delta_{\alpha}^{\mu} T_{\beta}^{\beta v}-\delta_{\alpha}^{v} T_{\beta}^{\beta \mu}\right)$,

which is skew symmetric in the last two indices. Similar to the $f(R)$ theory one can take the action of $f(T)$ theory as

$\mathscr{L}\left(h^{a}{ }_{\mu}, \Phi_{A}\right)=\int|h|\left[\frac{M_{\mathrm{Pl}}^{2}}{2} f(T)+\mathscr{L}_{m}\left(\Phi_{A}\right)\right] \mathrm{d}^{4} x$,

where $M_{\mathrm{Pl}}$ is the reduced Planck mass, which is related to the gravitational constant $G$ by $M_{\mathrm{Pl}}=\sqrt{\hbar c / 8 \pi G}$. Assuming the units in which $G=c=\hbar=1$, in the above equation $|h|=$ $\sqrt{-g}$ and $\Phi_{A}$ are the matter fields. The variation of (7) w.r.t. the tetrad field $h^{a}{ }_{\mu}$ requires the following field equations [24]:

$$
\begin{aligned}
& S_{\mu}{ }^{\rho v} \partial_{\rho} T f_{T T}+\left[h^{-1} h^{a}{ }_{\mu} \partial_{\rho}\left(h h_{a}{ }^{\alpha} S_{\alpha}{ }^{\rho v}\right)-T_{\lambda \mu}^{\alpha} S_{\alpha}{ }^{\nu \lambda}\right] f_{T} \\
& -\frac{1}{4} \delta_{\mu}^{v} f=-4 \pi \Theta_{\mu}{ }^{\nu},
\end{aligned}
$$

where $f \equiv f(T), f_{T}=\frac{\partial f(T)}{\partial T}, f_{T T}=\frac{\partial^{2} f(T)}{\partial T^{2}}$, and $\Theta_{\mu}{ }^{\nu}$ is the energy-momentum tensor.

\section{Cosmological modifications of $f(T)$}

We first assume that our universe is an isotropic and homogeneous, which directly gives rise to the tetrad field given by Robertson [61]. This can be written in spherical polar coordinate $(t, r, \theta, \phi)$ as follows:

$$
\left(h_{a}{ }^{\mu}\right)=\left(\begin{array}{llll}
1 & 0 & 0 & 0 \\
0 & \frac{L_{1} \sin \theta \cos \phi}{4 a(t)} & \frac{L_{2} \cos \theta \cos \phi-4 r \sqrt{k} \sin \phi}{4 r a(t)} & -\frac{L_{2} \sin \phi+4 r \sqrt{k} \cos \theta \cos \phi}{4 r a(t) \sin \theta} \\
0 & \frac{L_{1} \sin \theta \sin \phi}{4 a(t)} & \frac{L_{2} \cos \theta \sin \phi+4 r \sqrt{k} \cos \phi}{4 r a(t)} & \frac{L_{2} \cos \phi-4 r \sqrt{k} \cos \theta \sin \phi}{4 r a(t) \sin \theta} \\
0 & \frac{L_{1} \cos \theta}{4 a(t)} & \frac{-L_{2} \sin \theta}{4 r a(t)} & \frac{\sqrt{k}}{a(t)}
\end{array}\right),
$$

where $a(t)$ is the scale factor, $L_{1}=4+k r^{2}$, and $L_{2}=$ $4-k r^{2}$. The tetrad (9) has the same metric as the FRW metric,

$\mathrm{d} s^{2}=\mathrm{d} t^{2}-a^{2}(t)\left[\frac{\mathrm{d} r^{2}}{1-\frac{1}{4} k r^{2}}+r^{2} \mathrm{~d} \theta^{2}+r^{2} \sin ^{2}(\theta) \mathrm{d} \phi^{2}\right]$.

\subsection{Modified Friedmann equations}

Applying the $f(T)$ field equations (8) to the FRW universe (9), assuming an isotropic perfect fluid the energymomentum tensor takes the form $\Theta_{\mu}{ }^{v}=\operatorname{diag}(\rho,-p$, $-p,-p)$. The $f(T)$ field equations (8) read

$$
\begin{aligned}
\rho= & \Theta_{0}^{0}=\frac{1}{16 \pi}\left(f+12 H^{2} f_{T}\right), \\
p= & \Theta_{(\alpha)}(\alpha) \\
= & -\frac{1}{16 \pi}\left[\left(f+12 H^{2} f_{T}\right)+4 \dot{H}\left(f_{T}-12 H^{2} f_{T T}\right)\right. \\
& \left.-\frac{4 k}{a^{2}}\left(f_{T}+12 H^{2} f_{T T}\right)\right]
\end{aligned}
$$

where $(\alpha)$ identifies the spatial coordinate running from 1 , $\ldots, 3$ and $H(:=\dot{a} / a)$ is the Hubble parameter. The dot denotes the derivative w.r.t. cosmic time $t$. Equations (10) and (11) are the modified Friedmann equations in the $f(T)$ gravity. Substituting from the vierbein (9) into (5), we get the torsion scalar

$$
\begin{aligned}
T & =\frac{6 k-6 \dot{a}^{2}}{a^{2}} \\
& =-6 H^{2}\left(1+\Omega_{k}\right),
\end{aligned}
$$

where $\Omega_{k}:=-\frac{k}{a^{2} H^{2}}$ is called the curvature density parameter. In the above, the modified $f(T)$ Friedmann equations might show interesting results, since the first round bracket in (11) is the additive inverse of (10). This provides a good chance to hunt a cosmological constant-like matter perfectly, if the extra terms of (11) vanish. However, these terms enable evolutions away from the cosmological constant. By a careful look at (11) we find that these extra quantities can be split into a free $k$ term and a $k$ dependent term. ${ }^{2}$ Taking the SFU assumption leads to the disappearance of the last term in (11); then the chance for hunting cosmological constant-like DE occurs by taking the highly restrictive condition $\dot{H}=0$ or by taking its coefficient $f_{T}-12 H^{2} f_{T T}=0$. Since we are dealing within the SFU framework, the teleparallel torsion scalar (12) can be related to the Hubble parameter by $T=-6 H^{2}$. Only the SFU identifies a particular class of $f(T) \propto \sqrt{T}$. In this article we take a different path allowing evolution away from the cosmological constant without assuming spatial flatness, but enforcing the evolution to behave as in a flat-like model.

\footnotetext{
${ }^{2}$ One should note that $f(T)$ may contain $k$ dependent quantities.
} 


\subsection{Searching for a flat like universe}

Most cosmological models assume SFU models seeking for a perfect match with cosmological observations. As a matter of fact, taking the spatial flatness as a firm prediction of inflation is not quite accurate. It is easy to show that the smallness of $\Omega_{k}$ can be a reflection of $k \ll a^{2} H^{2}$ as expected for an early universe. It has been shown that even with a relatively large curvature parameter one can gain all advantages of inflation. On the other hand, it has been shown that the curvature density parameter average is $\left|\Omega_{k}\right| \lesssim 0.15$ at $1 \sigma$ confidence [62]. Moreover, it has been shown that the so-called modified growth parameters are correlated with the curvature density parameter with a recognizable deviation at $\left|\Omega_{k}\right| \geq 0.05$, which leads some to conclude that the spatial curvature must be included in the analysis with other cosmological parameters $[46,48,63,64]$. Recalling the $f(T)$ modified Friedmann equations (10) and (11), we allow the fluid to evolve away from the cosmological constant-like fluid regardless of the value of $k$. Instead of taking the constraint of the SFU model, we assume the vanishing of the coefficient of $k$ in (11), so that

$a^{2} f_{T}+12 \dot{a}^{2} f_{T T}=0$.

The FLU model ansatz (13) identifies a hidden special class of $f(T)$ gravity theories which cannot be covered by taking the SFU model. The solution of (13) is not easy in nonflat models. Also, it is worth to mention that this treatment cannot be applied in the TEGR theory, i.e. $f(T)=T$, since the coefficient of $k$ in (11) is a constant. So it would not provide us with a condition similar to (13) for examining a FLU. In this case one is obliged to assume global spatial flatness by putting $k=0$ to study the flat universe model. Thus we expect that the FLU treatment enables one to study gravity beyond the TEGR or GR domains. As $f(T)$ in FRW spacetime is a function of time $f(T \rightarrow t)$, one easily can show that

$f_{T}=\dot{f} / \dot{T}, \quad f_{T T}=(\dot{T} \ddot{f}-\ddot{T} \dot{f}) / \dot{T}^{3}$.

Substituting from (14) into (13), we then solve to $f(T \rightarrow t)$ to get

$f(t)=\Lambda+\lambda \int e^{\int \frac{k^{2}+\left(3 \ddot{a} a-5 \dot{a}^{2}\right) k+2 \ddot{a}^{2} a^{2}+4 \dot{a}^{4}-7 \dot{a}^{2} \ddot{a} a+\dot{a} \dddot{a} a^{2}}{\dot{a} a\left(\ddot{a} a-\dot{a}^{2}+k\right)} \mathrm{d} t} \mathrm{~d} t$,

where $\Lambda$ and $\lambda$ are integration constants. We later show that the constant $\Lambda$ can be perfectly interpreted as a cosmological constant. In order to reduce the dependence on $k$, we follow the same ansatz (13) by taking a vanishing value of the coefficient of $k$. So, in the above equation, we take

$3 \ddot{a} a-5 \dot{a}^{2}=0$, and by solving for $a(t)$ we get the scale factor

$a(t)=a_{0}\left[\frac{3}{3-2 H_{0}\left(t-t_{0}\right)}\right]^{3 / 2}$,

where $a_{0}$ is an arbitrary constant of integration, with the initial condition $H_{0}=H\left(t_{0}\right)$. One should mention that the scale factor is independent of the choice of $k$. On the other hand, we do not expect or accept that the three world models $k=0, \pm 1$ to be completely in coincidence; then the function $f(T \rightarrow t)$ should depend on the choice of $k$ in its final form. Substituting (17) into (12) we get the torsion scalar

$T(t)=\frac{6 k\left[3-2 H_{0}\left(t-t_{0}\right)\right]^{5}-1458 a_{0}^{2} H_{0}^{2}}{27 a_{0}^{2}\left[3-2 H_{0}\left(t-t_{0}\right)\right]^{2}}$.

Substituting from (17) into (15) and expanding the exponential around $t=0, f(T)$ is obtained as a function of $t$ as

$f(t)=\sum_{n=0}^{\infty} c_{n} t^{n}$

where $c_{n}$ are constant coefficients consisting of the set of constants $\left\{k, a_{0}, t_{0}, H_{0}, \lambda, \Lambda\right\}$. The zeroth term of (19) derives the Friedmann equations (10) and (11) to produce the cosmological constant DE, so it fixes the value of the coefficient $c_{0}$ to the cosmological constant. We give examples of the $c_{n}$ coefficients: $c_{0}=\Lambda, c_{1}=-2 \tau_{0} H_{0} \lambda k-\frac{81 a_{0}^{2} \lambda}{8 \tau_{0}^{4} H_{0}^{2}}$, $c_{2}=-\frac{81 a_{0}^{2} \lambda}{4 \tau_{0}^{5} H_{0}^{2}}-\frac{16 \tau_{0}^{5} H_{0}^{4} \lambda k^{2}}{81 a_{0}^{2}}, \ldots$ etc., where $\tau_{0}=t_{0}+\frac{3}{2 H_{0}}$. The higher orders of the expansion in (19) produce the evolution away from the cosmological constant. Equation (18) enables us to write the time mathematically in terms of the torsion scalar, i.e. $t=\tau_{0}+\frac{3 \sqrt{6} / 2}{\sqrt{-T}}+O\left(\frac{1}{T^{3}}\right)$; then we reexpress (19) in terms of the torsion scalar as an inverse power law of $T$ as $^{3}$

$f(T) \propto \sum_{n=0}^{\infty} \frac{\alpha_{n}}{\sqrt{-T^{n}}}$,

where $\alpha_{n}$ are known constant coefficients consisting of the set of constants similar to the coefficients $c_{n}$. The above expression shows that $f(T)$ does not reduce to TEGR as expected so that it describes non-ordinary matter. Accordingly, we cannot assume a fixed equation of state (EoS) as in the classical cases. But it is more convenient to consider the case of the time dependent EoS corresponding to the contribution of the dynamical evolution of the $f(T)$ to the density and pressure. We summarize the FLU model as follows:

(a) In the GR theory, a fixed value of EoS parameter, $\omega:=$ $p(a(t)) / \rho(a(t))$, is entered into the Friedmann equa-

\footnotetext{
3 We give the $f(T)$ in terms of the torsion scalar to explore the $f(T)$ in its usual form. But all the calculations in this work are performed using the time dependent form (19).
} 
tions as an input; then the scale factor $a(t)$ is obtained as an output.

(b) In the $f(T)$ theories, a fixed EoS parameter in addition to a scale factor $a(t)$ is entered into the Friedmann equations as inputs; then an $f(T)$ is obtained as an output.

(c) In this work, which is governed by the $f(T)$ framework, we introduce two conditions (the FLU model assumptions (15) and (16)) as inputs; then we get a scale factor $a(t), f(T)$, and a dynamical EoS parameter $\omega(t)$ as outputs.

In case (c), the FLU constrains the scale factor $a(t)$ and $f(T)$ by the model assumptions. These assumptions allow the density and pressure to be reformulated accordingly as functions of time. So it is not convenient to add an extra condition, e.g. assuming a fixed EoS. Whereas the EoS in the FLU model should be treated as a time dependent output parameter, i.e. $\omega(t)=p(t) / \rho(t)$, this guarantees a consistent system of Friedmann equations. Actually, the only thing that we have to worry about, in $f(T)$ of cosmological applications, is obtaining compatible $a(t)$ and $f(T)$ [46]. It is worth to mention that the fine tuning problem facing DE models with a constant equation of state can be alleviated if we assume that the EoS is time dependent, e.g. if we have quintessence models [65]. So one should think of this model as a quintessence model rather than a classical cosmological model.

\section{Cosmic evolution}

In this section, we perform a cosmological study of the FLU model to examine its capability to obtain the cosmic evolution. In addition, we study its consistency with the Friedmann equations. The FRW spacetime that is governed by $f(T)$ gravity can be determined by a scale factor $a(t)$ and an $f(T)$ form. As we mentioned before the scale factor (17) is independent of $k$, while the $f(t)$, namely (19), depends on $k$ as it should. This allows one to study different evolution scenarios according to the choice of $k$, assuming that the FRW universe is filled with a single-fluid component given by (10) and (11); we examine the cosmic evolution as follows.

\subsection{The large Hubble spacetime}

We study the large $H$-regime, i.e. at early times of the universe. The Hubble parameter corresponds to the scale factor (17) given by

$$
H=\frac{3 H_{0}}{3-2 H_{0}\left(t-t_{0}\right)},
$$

where the modified Friedmann equations are symmetric under $a \rightarrow-a$. We easily find that the universe is always accelerating as the deceleration parameter $q:=$ $-\frac{a \ddot{a}}{\dot{a}^{2}}=-5 / 3$. Nevertheless, the matter density (10) of this theory in the large $H$-spacetime is given as $\rho \rightarrow$ $\frac{1}{16 \pi}\left(\Lambda+\frac{81 a_{0}^{2} H_{0}^{2} \lambda}{\left(3+2 H_{0} t_{0}\right)^{3}}\right)$, which agrees with the predictions of the vacuum density with a correction term. This is consistent with the inflationary universe scenario at this stage.

\subsection{Single-fluid equation of state}

In this $f(T)$ theory, we assume only a single-fluid component to describe the matter of the universe. As we mentioned in Sect.3.2, there is a good chance to hunt negative pressure matter in the $f(T)$ framework with expected deviation from the cosmological constant behavior. We now examine the nature of this single-fluid component and its possible evolution. Using (10) and (11), we get a time dependent EoS parameter $(\omega:=p / \rho)$. The dynamical behavior shows a similar asymptotic behavior regardless of the universe being flat or not. It is only affected by the order of expansion of (19). We need to mention that the $f_{n}(t)=c_{0}+c_{1} t+c_{2} t^{2}+\cdots+c_{n} t^{n}$ shows that the leading term is $f_{0}(t)=c_{0}=\Lambda$, producing an EoS as a constant function of time $\omega(t)=-1$, which describes the cosmological constant DE perfectly. However, the value of $\Lambda$ is not necessarily large, as we will see later. We choose initial conditions to fit with the early universe, by taking a tiny initial scale factor combined with a large initial Hubble constant at Planck time $t_{0}=t_{\mathrm{Pl}}$. When the first-order correction is taken into consideration, the EoS approaches $\omega \rightarrow-7 / 9$ as $t \rightarrow t_{\mathrm{f}}$, where $t_{\mathrm{f}} \gg t_{\mathrm{Pl}}$ is a time large enough for the EoS to get its final steady phase. The second-order correction allows the EoS to evolve up to $\omega \rightarrow-5 / 9$ as $t \rightarrow t_{\mathrm{f}}$. We conclude that the series of the $f(t)$ up to the second-order correction does not allow for the EoS crossover $\omega=0$ at any time. These cases of $n=0,1,2$ are useful to describe the very early universe.

We give the model with $n=3$ more attention as it produces excellent agreement with the acceptable cosmic evolution scenario; see the plots of Fig. 1. Although we used a single-fluid component, its dynamical evolution allows the EoS parameter initially to start from $\omega<-1$ (phantom). The evolution of the EoS shows almost the same behavior in the three models. Then it evolves to pressureless cold dark matter $(\mathrm{CDM})(\omega=0)$, radiation $(\omega=1 / 3)$, and possibly stiff matter $(\omega=1)$, while it shows a quintessence fate $\omega \rightarrow-1 / 3$ as $t \rightarrow t_{\mathrm{f}}$ in all models. This shows the unique origin of early and late cosmic accelerating expansion. So we find that the third-order correction is the most physically relevant scenario. In the cases of $n \geq 4$, we have a physical motivation to study the series up to $n=9$ only as the EoS of higher order produces $\omega>1$ asymptotically, which represents unknown matter so far.

We find that the EoS asymptotic behavior increases by a quantized value $\frac{2}{9}$ as $\left\{-1,-\frac{7}{9},-\frac{5}{9}, \ldots\right\}$ as the $n$th order increases by unity in the series (19). 


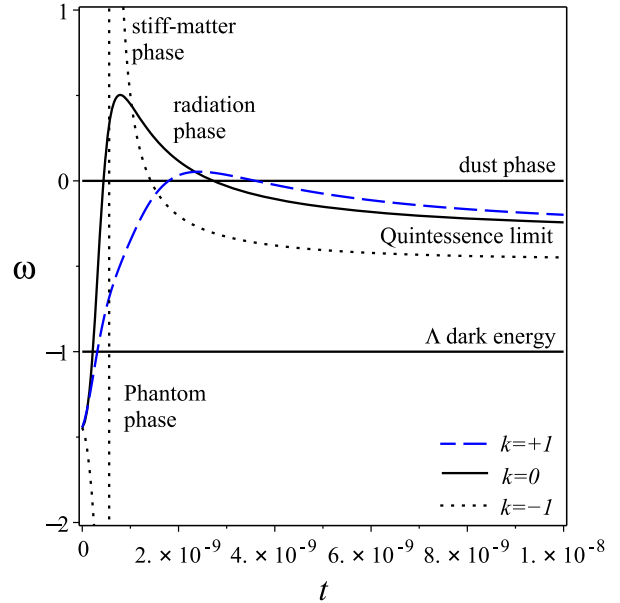

Fig. 1 The EoS up to $O\left(t^{3}\right)$ evolution: the solid line represents the evolution in the flat space, while the dot and dash lines represent the evolution in the open and closed models, respectively. The plots show almost the same initial phantom state and the same quintessence behavior fate, which is in agreement with the expected evolution in the FLU model. The initial conditions are taken to fit with an early time phase as $a_{0}=10^{-9}, H_{0}=10^{9} s^{-1}, t_{0}=t_{\mathrm{Pl}}=10^{-44} \mathrm{~s}, \Lambda=10^{-30} \mathrm{~s}^{-2}$, and $\lambda=3$

It is worth to mention that the FLU model shares with the quintessence models an important result, a dynamical EoS. In addition, the theory predicts similar evolutions at early time in the three models $k=0, \pm 1$. Moreover, it forces the flat/non-flat models to have identical behaviors at a later time. Thus, the FLU model succeeds in describing an effectively flat solution without imposing a globally vanishing sectional curvature by taking $k=0$.

\subsection{Conservative universe}

As mentioned in the introduction of the present section we extract a scale factor (17) and an $f(T)(19)$ as solutions of two assumptions (15) and (16) of the FLU model, not directly from the Friedmann equations as usual. We examine the validity of the Friedmann equations for this model. Actually, for the case of the cosmological constant with a fixed EoS parameter $(\omega=-1)$ that is required to describe the accelerating universe, one cannot get an evolutionary scenario for this dark component. In addition, its continuity equation,

$\dot{\rho}+3 H(\rho+p)=0$,

shows that $\dot{\rho}=0$, i.e. the density is constant. Nevertheless, the density should decrease as the universe expands. This conflict implies a continuous creation of matter in order to keep the density of the expanding universe constant. This case is similar to the steady state cosmology which breaks the conservation principle. This problem vanishes as a consequence of assuming a dynamical EoS as in our case with the FLU model. In this $f(T)$ theory, we use one fluid compo- nent to describe the evolution of the universe, with a negative pressure matter dominating most of the different epochs during the early time evolution. Moreover, we get a conservative universe. This can be obtained by substituting from (17) and (19) into (10) and (11), which shows that the continuity equation is always verified up to any order of expansion of (19).

We summarize the results of Sect. 4 as follows: The FLU model (i) is consistent with an inflationary scenario universe, (ii) has a dynamical EoS parameter which allows for cosmic evolution, and (iii) satisfies the continuity equation of Friedmann. It is well known that the scalar field plays a key role in the quintessence models to interpret the inflation stage at an early universe. On the other hand, the torsion plays the main role in teleparallel geometry. We next consider the role of the torsion as an added value quality of the spacetime when formed by a scalar field and its role at the time of the early universe.

\section{Torsion potential}

In order to get an idea of the torsion potential, we first discuss the physical meaning of the connection coefficients as the displacement field. So we use (3) to reexpress the Weitzenböck connection (1) as

$\Gamma_{\nu \rho}^{\mu}=\stackrel{\circ}{\Gamma}_{\nu \rho}^{\mu}+K_{\nu \rho}^{\mu}$.

By careful looking at the above expression of the new displacement field, it consists of two terms. The first is the LeviCivita connection which consists of the gravitational potential (metric coefficients, $g_{\mu \nu}$ ) and its first derivatives w.r.t. the coordinates. Here the second term is the contortion, which consists of the tetrad field and its first derivatives w.r.t. the coordinates. In this sense we find that the first term contributes to the displacement field as the usual attractive force of gravity, while the second term contributes as a force too. The equation of motion tells us that this force is repulsive [66]. Now we can see how teleparallel geometry adds a new quality (torsion or contortion) to the spacetime, allowing the repulsive side of gravity to show up.

\subsection{Torsion potential of a scalar field}

We consider here the physical approach to construct the torsion from a scalar field $\varphi(x)$. In view of the above discussion, we can treat the contortion in the Weitzenböck connection (23) as a force. Accordingly, one is required to construct the contortion (or torsion) from a tensor and its first-order derivatives. This tensor now plays the role of the potential of the torsion. We follow the approach that has been proposed by [67], by introducing 16 fields $t_{a}^{\mu}$, which are called the "torsion potential". These fields form a quadruplet of basis vectors, so we write the following linear transformation: 
$h_{a}=t^{\mu}{ }_{a} \partial_{\mu}, \quad h^{a}=t^{a}{ }_{\mu} \mathrm{d} x^{\mu}$,

the torsion potential $t_{a}^{\mu}$ and its inverse satisfying the conditions:

$t=\operatorname{det}\left(t^{\mu}{ }_{a}\right) \neq 0, \quad t^{\mu}{ }_{a} t^{a}{ }_{\nu}=\delta_{v}^{\mu}, \quad t^{\mu}{ }_{a} t^{b}{ }_{\mu}=\delta_{a}^{b}$.

Finally, this enables us to express the torsion as [67]

$T_{\mu \nu}^{\alpha}=t^{\alpha}{ }_{a}\left(\partial_{\mu} t^{a}{ }_{\nu}-\partial_{\nu} t_{\mu}^{a}\right)$.

The torsion potential $t^{\mu}{ }_{a}$ can be formed by physical scalar, vector or tensor fields. This may be of great interest for physical applications. Now we take the case when the torsion potential is formed by a scalar field $\varphi(x)$ by taking

$t^{a}{ }_{\mu}=\delta_{\mu}^{a} e^{\sqrt{3 / 2} \varphi}, \quad t^{\mu}{ }_{a}=\delta_{a}^{\mu} e^{-\sqrt{3 / 2} \varphi}$,

where $\varphi$ is a non-vanishing scalar field. Then the torsion and the contortion (3) can be reexpressed, respectively, as

$T^{\alpha}{ }_{\mu \nu}=\sqrt{3 / 2}\left(\delta_{\nu}^{\alpha} \partial_{\mu} \varphi-\delta_{\mu}^{\alpha} \partial_{\nu} \varphi\right)$,

$K^{\mu \nu}{ }_{\alpha}=\sqrt{3 / 2}\left(\delta_{\alpha}^{\nu} \partial^{\mu} \varphi-\delta_{\alpha}^{\mu} \partial^{\mu} \varphi\right)$,

where $\partial^{\mu} \varphi=g^{\mu \alpha} \partial_{\alpha} \varphi$. The above expressions have been used in order to formulate a theory of electromagnetism minimally coupled to torsion and satisfying gauge invariance and the minimal coupling principles [68-71]. Using (6), (28), and (29), the teleparallel torsion scalar (5) can be written in terms of the scalar field $\varphi$ as

$T=-9 \partial_{\mu} \varphi \partial^{\mu} \varphi$.

The above treatment shows that the torsion acquires dynamical properties and it propagates through space. It is worth to mention that the energy-momentum tensor of a free scalar field represents a source of the dynamical torsion in the teleparallel description of gravity [72], which is not in agreement with the common belief that the spin matter only can produce torsion [15]. One should mention here that the same results can be obtained by combining the conformal transformation of the tetrad fields $h^{\mu}{ }_{a} \rightarrow e^{\varphi} h^{\mu}{ }_{a}$ and $h^{a}{ }_{\mu} \rightarrow e^{-\varphi} h^{a}{ }_{\mu}$, in addition to the so-called Einstein $\lambda$ transformation (projective transformation) of the connection coefficients $[73,74]$ as

$\Gamma_{\mu \nu}^{\alpha} \rightarrow \Gamma_{\mu \nu}^{\alpha}-\delta_{\nu}^{\alpha} \partial_{\mu} \varphi$.

Actually, this approach, indeed, has a geometrical framework as well. Where the connection is assumed to be a semisymmetric one, this case has been studied by many authors, cf. [75].

\subsection{Gravitational quintessence model}

It is well known that cosmic inflation is powered by a scalar field $\varphi$. On the other hand, the cosmological applications of $f(T)$ gravity show strong evidence of cosmic inflation. So there must be a link between these two descriptions, and we see that Eq. (30) provides this link as the teleparallel torsion scalar appears as a gradient of a scalar field. So it acquires dynamical properties and propagates through space. Also, Eq. (30) enables us to map the density and pressure contributions in the Friedmann equations into the scalar field $\left(\rho \rightarrow \rho_{\varphi}, p \rightarrow p_{\varphi}\right.$ ). This leads us to reformulate the Friedmann equations of the torsion contribution as an inflationary background in terms of the scalar field $\varphi$. As mentioned before the FLU model provides $f(T) \propto \sum \frac{1}{\sqrt{-T^{n}}}$ and does not reduce to TEGR theory of ordinary matter. This is in agreement with the inflationary epoch where the matter contribution can be negligible. Accordingly, we consider the Lagrangian density of a homogeneous (real) scalar field $\varphi$ in a potential $V(\varphi)$,

$\mathscr{L}_{\varphi}=\frac{1}{2} \partial_{\mu} \varphi \partial^{\mu} \varphi-V(\varphi)$,

where the first expression in (32) represents the kinetic term of the scalar field, as usual, while $V(\varphi)$ represents the potential of the scalar field. The variation of the action w.r.t. the metric $g_{\mu \nu}$ enables one to define the energy-momentum tensor as

$\mathscr{T}^{\mu \nu}=\frac{1}{2} \partial^{\mu} \varphi \partial^{\nu} \varphi-g^{\mu \nu} \mathscr{L}_{\varphi}$.

The variation w.r.t. the scalar field leads to the scalar field density and pressure, respectively, as follows:

$\rho_{\varphi}=\frac{1}{2} \dot{\varphi}^{2}+V(\varphi), \quad p_{\varphi}=\frac{1}{2} \dot{\varphi}^{2}-V(\varphi)$.

The Friedmann equation (10) of the non-flat models in the absence of matter becomes

$H^{2}=\frac{8 \pi}{3}\left(\frac{1}{2} \dot{\varphi}^{2}+V(\varphi)\right)-\frac{k}{a^{2}}$.

Using (34) it is an easy task to show that the continuity equation (22) reduces to the Klein-Gordon equation of a homogeneous scalar field in the expanding FRW universe,

$\ddot{\varphi}+3 H \dot{\varphi}+V^{\prime}(\varphi)=0$,

where the prime denotes the derivative w.r.t. the scalar field $\varphi$. In conclusion, Eq. (30) enables us to define a scalar field sensitive to the vierbein field, i.e. the spacetime symmetry. In addition, Eq. (34) enables us to evaluate an effective potential from the adopted $f(T)$ gravity theory. The mapping from the torsion contribution to the scalar field fulfills the Friedmann and Klein-Gordon equations. So the treatment meets the requirements to reformulate the torsion contribution in terms of a scalar field without attempting a conformal transformation. 


\subsection{Generalized Starobinsky potential by $f(T)$}

In the following treatment, we take the simple case of the flat space universe $k=0$ in order to compare the obtained results to the standard treatment of the scalar field theory in cosmology. Using (18), (30) we get

$\dot{\varphi}^{2}=\frac{3 / 2}{\left(t-\tau_{0}\right)^{2}}$,

where $\tau_{0}$ is as given in Sect. 3.2. Integrating the above equation we write the scalar field $\varphi$ as

$\varphi=\varphi_{0} \pm \sqrt{6} / 2 \ln \left(t-\tau_{0}\right)$,

and $\varphi_{0}=\varphi\left(t_{0}\right)$ is a constant of integration. The above equation allows one to express the time $t$ mathematically in terms of the scalar field $\varphi$. So all the dynamical expressions can be expressed in terms of the scalar field.

The relation between the scalar fields $T$ and $\varphi$ has to be investigated. Using (18) and (38) we can express the torsion scalar field $T$ in terms of the scalar field $\varphi$ as

$T(\varphi)=-\frac{27}{2} e^{ \pm 2 \sqrt{2 / 3}\left(\varphi-\varphi_{0}\right)}$.

As is well known taking the strong coupling condition the inflaton field is related to the canonical scalar field $\Omega$ by [76]

$\varphi= \pm \sqrt{\frac{3}{2}} \log \Omega$

Comparing the above equation with the teleparallel torsion (39) we get the transformation $T=-\xi \Omega^{2}$ where the constant $\varphi_{0}$ can be absorbed in the coefficient $\xi$. This indicates that the teleparallel torsion scalar might play a role similar to the canonical scalar field normally used in scalar-tensor theories of gravitation. Also, using (17) and (19), the pressure (11) can be reexpressed in terms of the scalar field $\varphi$ as

$p_{\varphi}=-\frac{\Lambda}{16 \pi}-\lambda \sum_{n=0} \beta_{n} e^{-n \sqrt{2 / 3}\left(\varphi-\varphi_{0}\right)}$,

where the $\beta_{n}$ are known constant coefficients. Similarly, we can evaluate the scalar field density (34). With simple calculations one can find the continuity equation of the scalar field still to be valid. Substituting (37) and (41) into (34), we evaluate the potential of the scalar field $\varphi$ as

$V(\varphi)=V_{0}+\lambda \sum_{n=0} \beta_{n} e^{-n \sqrt{2 / 3}\left(\varphi-\varphi_{0}\right)}$,

where $V_{0}=\frac{\Lambda}{16 \pi}+\frac{3}{4} e^{2 \sqrt{2 / 3}\left(\varphi-\varphi_{0}\right)}$. The higher-order contributions in (42) are due to the order of expansion of $f(T)$, which allows for different potential types of slow-roll inflation models. So we find that both kinetic and potential energies are formed from the teleparallel torsion and $f(T)$ gravity, respectively. It is well known that the scalar field potential of the cosmic inflation has many different forms according to the assumed model, e.g. polynomial chaotic, power law, natural, intermediate, etc. In this work, we provide another approach to construct the potential from the $f(T)$ gravity theory. According to the order of expansion of the potential (42), this gives either a Starobinsky-like inflation model [77], where the potential blows up at $\varphi<0$ and inflation can occur only at $\varphi>0$, or it gives a quadratic-like inflation model, where the potential allows inflation to occur at both $\varphi<0$ and $\varphi>0$. This will be discussed in more detail below.

\subsection{Classified torsion scalar potentials}

In the previous section we applied a new technique to induce the scalar potential (42) by the $f(T)$ gravity (19). In this theory we obtained a power series potential of $e^{-\sqrt{2 / 3} \varphi}$ where $\varphi$ represents the inflaton field. So the theory may cover different classes of inflationary models. We next examine the potentials correspond to the order of the expansion of (19). In this way, and with the help of the evaluated EoS induced by the fluid (10) and (11), we might be able to compare these potentials to the already known inflaton potentials. The triple (19), EoS and (42) would enable us to give a physical classification of these potentials.

\subsubsection{The $V_{0}$-model}

The modified Friedmann equations (10) and (11) of the $f(T)$ gravity theories pay attention to the choice of $f(T)=$ const. as it acts perfectly as the cosmological constant. This is exactly the case here, when assuming the zeroth order of (19) solution. We first evaluate the above mentioned triple as

$f_{0}(T)=\Lambda$

$\omega_{0}=-1$,

$V_{0}=\frac{\Lambda}{16 \pi}+\frac{3}{4} e^{2 \sqrt{2 / 3}\left(\varphi-\varphi_{0}\right)}$,

where $-\infty<\varphi<\infty$. It is clear that $f_{0}(T)$ acts perfectly as a cosmological constant DE ( $\triangle \mathrm{DE})$ with $\operatorname{EoS} \omega=-1$. A generic potential of this type can be obtained by adding a constant to exponential potential (power law inflation); see Fig. 2a. We will see later that this potential pattern is recommended to describe the $E$ and $B$ modes of the power spectrum polarization. The first term in (43) is the vacuum energy density (cosmological constant) of the false vacuum spacetime, while the second term associated to the vacuum potential represents a phase transition potential dragging the universe away from the false vacuum $(\varphi=0$ state $)$ to a true vacuum ( $\varphi \neq 0$ state $)$ at its minimum effective potential. However, the EoS still obeys $\omega_{0}=-1$ during the whole stage. Now, we investigate the phase transition potential in (43). Using the relation (40) we write the $V_{0}$ potential in terms of the canonical scalar field as 


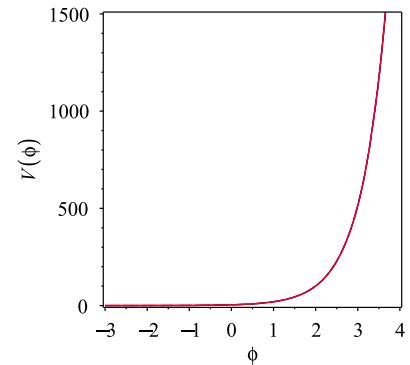

(a) $n=0$

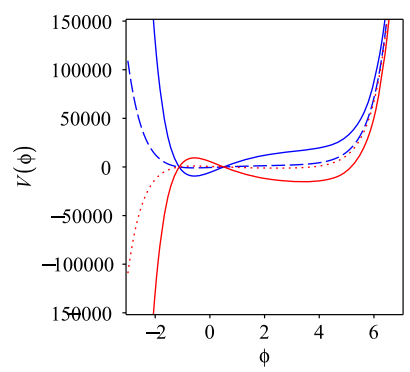

(c) $n=2$

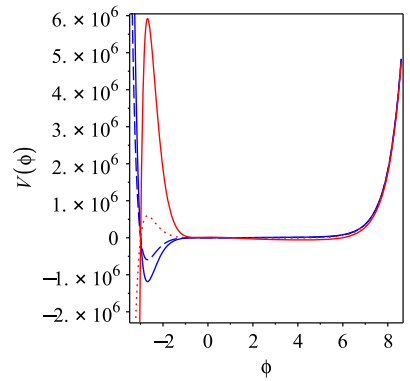

(e) $n=4$

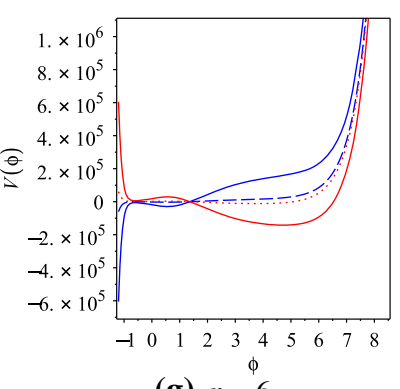

(g) $n=6$

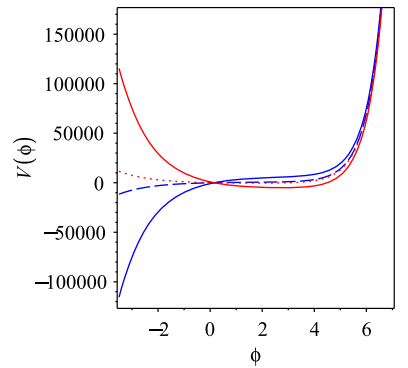

(b) $n=1$

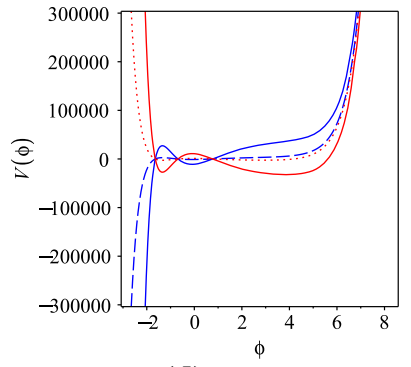

(d) $n=3$

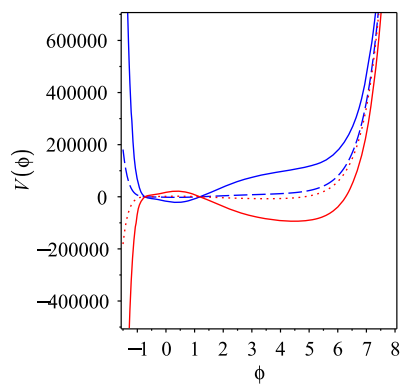

(f) $n=5$

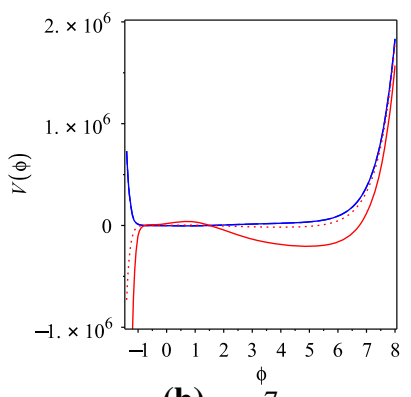

(h) $n=7$
Fig. 2 Schematic plots of the scalar field potentials according to the order of expansion of the $f(T)$ function. The set of parameters $\left\{t_{0}, a_{0}, H_{0}, \Lambda\right\}$ are taken as in Fig. 1. The blue solid lines are for $\lambda>1$, the blue dash lines are for $0<\lambda<1$, the red dot lines are for $0>\lambda>-1$, and the red solid lines are for $\lambda<-1$

$V_{0} \propto 1 / \Omega^{2}$,

which gives an inverse square law potential. This type of potentials represents the original class of quintessence fields. Indeed, the previous expression shows a minimum effective potential only at $\Omega>0$ or $\Omega<0$ with a potential barrier at $\Omega=0$, while (43) allows the full range $-\infty<\varphi<\infty$. But as the inflation occurs only in a single plateau, the two expression are identical at $\varphi>0$.

\subsubsection{The $V_{1}$-model}

We take the expansions up to the first order giving the triple

$f_{1}(T)=\Lambda-\frac{a_{0}^{2} \lambda}{H_{0}^{2} \tau_{0}^{3}}\left[\frac{81}{8}+\frac{243 \sqrt{6}}{16 \tau_{0} \sqrt{-T}}\right]$,

$\lim _{t \rightarrow t_{\mathrm{f}}} \omega_{1}=-7 / 9$,

$V_{1}=V_{0}-\frac{a_{0}^{2} \lambda}{H_{0}^{2} \tau_{0}^{3}}\left[\frac{81}{128 \pi}+\frac{63 / \tau_{0}}{64 \pi} e^{-\sqrt{2 / 3}\left(\varphi-\varphi_{0}\right)}\right]$.

We have $f_{1}(T) \propto \frac{1}{\sqrt{-T}}$, which is usually used to identify the CDM. In this model, the additional terms of the potential $V_{1}$, given by (46), contribute to a decrease of the potential relative to the kinetic energy. This allows the EoS to evolve above the $\Lambda \mathrm{DE}$ value by $2 / 9$, i.e. $\omega_{1} \rightarrow-7 / 9$, which is in agreement with the previous results of Sect.4.2. Moreover, the potential $V_{1}$ reduces to $V_{0}$ where $\lambda=0$. However, the non-vanishing values of $\lambda$ show interesting patterns; their plots appear in Fig. 2b. They predict similar behaviors at $\varphi>0$ where the potentials are nearly flat at the false vacuum $(\varphi=0)$ and the system slowly rolls to its effective minimum at the true vacuum $(\varphi \neq 0)$. At $\varphi<0$, the potentials predict different behaviors according to the value of $\lambda$. At very small negative or positive values of $\lambda$ the potentials are nearly flat. The large positive $\lambda$ produces Starobinsky-like model where the potential blows up exponentially. Nevertheless, the large negative $\lambda$ turns the potential to a quadratic-like model.

\subsubsection{The $V_{2}$-model}

With higher-order expansion we have these triple

$$
\begin{aligned}
f_{2}(T) & =\Lambda-\frac{a_{0}^{2} \lambda}{H_{0}^{2} \tau_{0}^{3}}\left[\frac{243}{8}+\frac{1215 \sqrt{6}}{16 \tau_{0} \sqrt{-T}}-\frac{2187}{8 \tau_{0}^{2} T}\right], \\
\lim _{t \rightarrow t_{\mathrm{f}}} \omega_{2} & =-5 / 9, \\
V_{2}= & V_{0}-\frac{a_{0}^{2} \lambda}{H_{0}^{2} \tau_{0}^{3}}\left[\frac{243}{128 \pi}+\frac{315}{64 \pi} e^{-\sqrt{2 / 3}\left(\varphi-\varphi_{0}\right)}\right. \\
+ & \left.\frac{135 / \tau_{0}}{64 \pi} e^{-2 \sqrt{2 / 3}\left(\varphi-\varphi_{0}\right)}\right] .
\end{aligned}
$$

This case is still in the quintessence range where $\omega_{2} \rightarrow$ $-5 / 9$. The potential $V_{2}$ reduces to $V_{0}$ at the limit $\lambda \rightarrow 0$ where the kinetic energy dominates over the potential. On the other hand, $V_{2}$ reduces to the original Starobinsky potential where the kinetic energy is negligible during the inflation. The Starobinsky pattern is clear in Fig. $2 \mathrm{c}$ in the case $\lambda>1$ where the potential is dominant. The non-vanishing values of $\lambda$ produce almost the same behavior at $\varphi>0$, while they produce different behaviors at $\varphi<0$. In contrast to the $V_{1}$, the large negative $\lambda$ allows the Starobinsky-like model to dominate the $\varphi<0$ epoch, while the large positive $\lambda$ turns the potential to a quadratic-like model; see Fig. 2c, at $\varphi<0$. 
Its plot shows two different minima allowing for inflation in both $|\varphi|>0$.

\subsubsection{The $V_{3}$-model}

We study one more case: where the triple are given by

$$
\begin{aligned}
& f_{3}(T)= \Lambda-\frac{a_{0}^{2} \lambda}{H_{0}^{2} \tau_{0}^{3}}\left[\frac{513}{8}+\frac{3645 \sqrt{6}}{16 \tau_{0} \sqrt{-T}}-\frac{6561}{4 \tau_{0}^{2} T}\right. \\
&\left.-\frac{10935 \sqrt{6}}{16 \tau_{0}^{3} \sqrt{-T^{3}}}\right] \\
& \lim _{t \rightarrow t_{\mathrm{f}}} \omega_{3}=-1 / 3 \\
& V_{3}=V_{0}-\frac{a_{0}^{2} \lambda}{H_{0}^{2} \tau_{0}^{3}}\left[\frac{513}{128 \pi}+\frac{945 / \tau_{0}}{64 \pi} e^{-\sqrt{2 / 3}\left(\varphi-\varphi_{0}\right)}\right. \\
&\left.+\frac{405 / \tau_{0}^{2}}{32 \pi} e^{-2 \sqrt{2 / 3}\left(\varphi-\varphi_{0}\right)}+\frac{45 / \tau_{0}^{3}}{16 \pi} e^{-3 \sqrt{2 / 3}\left(\varphi-\varphi_{0}\right)}\right] .
\end{aligned}
$$

The evolution of the EoS for this case has been studied in this work in Sect.4.2. The interest in this case is motivated by the study of the so-called "tracker field" when assuming the inflation ends at $\omega_{3} \rightarrow-1 / 3$ [78]. The potential $V_{3}$ reduces to $V_{0}$ when $\lambda$ vanishes, its nonvanishing values show general behaviors similar to $V_{1}$. Figure $2 \mathrm{~d}$ shows that, in particular for large negative $\lambda$, the false vacuum is separated by a broad barrier. However, the top of the barrier is quite flat. The decay of the false vacuum is followed by slow-roll inflation allowing a tunneling event from the high energy false vacuum. We find that this model fulfills the requirements of [79] to perform well fitting both the $E$-mode and the $B$ mode polarizations.

\subsubsection{The $V_{n \geq 4}$-models}

For the $V_{4}$-model we find it similar to $V_{2}$-model but with strong flat plateau at $\varphi>0$ of the false vacuum. At $\varphi<0$ the symmetry of negative and positive large values of $\lambda$ no longer valid; see the plots of Fig. 2e. For the $V_{n>4}$-models, the symmetry of large $\pm \lambda$ holds again but they alter their roles; see the plots of Fig. $2 \mathrm{f}-\mathrm{h}$. Moreover, the asymptotic EoS comes to increase by $2 / 9$ with a radiation limit for $\omega_{6}$ while $\omega_{9}$ gives stiff matter, and all higher-order expansions give unknown matter, so far, with $\omega>1$. In order to check the consistency of the results which are obtained by the $f(T)$ gravity and by the scalar field of the FRW model, we use Eqs. (37)-(42) to evaluate the dynamical EoS of the scalar field, $\omega_{\varphi}=p_{\varphi} / \rho_{\varphi}$, according to the order of expansion. The calculations show that the EoS of the $n=0$ model predicts a cosmological constant like $\operatorname{DE} \omega(\varphi)=-1$. The additional terms of the series of $e^{-n \sqrt{2 / 3}\left(\varphi-\varphi_{0}\right)}$ in (42) diminish the effective potential, gradually allowing the kinetic energy to show up effectively as the order of expansion increases, so that the EoS approaches different asymptotic values with the arithmetic sequence $\left\{-\frac{7}{9},-\frac{5}{9},-\frac{1}{3}, \ldots\right\}$, respectively. So the rushing of the EoS $\omega_{\varphi}$ toward $\omega_{\varphi}>-1$ is powered by the kinetic energy of the scalar field. When the higher orders of expansion of (42) are taken into consideration, the kinetic energy is enough to end the cosmic inflation, allowing the EoS to cross over $\omega_{\varphi}=0$ to enter a matter dominant epoch for the universe. It is clear that the scalar field analysis is in agreement with the results of the $f(T)$ treatment in Sect. 4.2.

Now turning back to the plots of Fig. 2, the overall picture shows similar behaviors for all the models at $\varphi>0$, while they interpolate between Starobinsky and polynomial potentials at $\varphi<0$ with different details as mentioned above. It is well known that the $B$-mode polarization excludes the small tensor-to-scalar ratio models such as the Starobinsky model [59]. In contrast, the Planck data restricts the tensor-to-scalar ratio to be small, so it excludes inflationary models such as large-field inflation models with a single monomial term $[57,58]$. In this theory, we find that the inflationary potential interpolates between these two different classes of inflation. These results lead us to investigate the inflationary parameters within this theory.

\section{Single-scalar field with double slowly rolling solutions}

We assume that the inflation epoch is dominated by the scalar field potential only. The slow-roll models define two parameters by

$\epsilon=\frac{1}{16 \pi}\left(\frac{V^{\prime}}{V}\right)^{2}, \quad \eta=\frac{1}{8 \pi}\left(\frac{V^{\prime \prime}}{V}\right)$.

These parameters are called slow-roll parameters. Consequently, the slow-roll inflation is valid where $\epsilon \ll 1$ and $|\eta| \ll 1$ when the potential is dominating. While the end of inflation is characterized by $\operatorname{Max}(\epsilon,|\eta|)=1$ as the kinetic term contribution becomes more effective. The slow-roll parameters define two observable parameters,

$r=16 \epsilon, \quad n_{s}=1-6 \epsilon+2 \eta$,

where $r$ and $n_{s}$ are called the tensor-to-scalar ratio and scalar tilt, respectively. Recent observations by Planck and BICEP2 measure almost the same scalar tilt parameters, $n_{s} \sim 0.96$. However, Planck puts an upper limit $r<0.11$, which supports models with small $r$, while BICEP2 sets a lower limit on the $r>0.2$, which supports inflationary models with large $r$. We devote this section to an investigation of the capability of the slow-roll models to match both Planck and BICEP2. 


\subsection{Construct a potential from Planck and BICEP2}

It is clear that Planck and BICEP2 observations agree on the scalar tilt parameter value $n_{s} \sim 0.963$, while they give different tensor-to-scalar ratios $r$. In order to construct a scalar potential performing Planck and BICEP2 data, we found that if the slow-roll parameters (55) satisfy the proportionality relation $\epsilon \propto \eta^{2}$, this gives a chance to find two values of $\eta$ performing the same $n_{s}$ but two different values of $\epsilon$. Consequently, we have two values of $r$. This can be achieved as follows: using (56) and the proportionality relation we have

$n_{s}=1-6\left(\epsilon=\kappa \eta^{2}\right)+2 \eta$,

i.e. $\eta^{ \pm}=\frac{1}{6 \kappa}\left(1 \pm \sqrt{1+6 \kappa\left(1-n_{s}\right)}\right)$,

where $\kappa$ is a constant coefficient and $\eta^{ \pm}$are due to the \pm discriminant. It is clear that there are possibly two different values of the parameter $\eta$ for a single-scalar tilt parameter $n_{s}$. Accordingly, we have $\epsilon=\kappa \eta^{2}=16 / r$, which provides double values of $r$ for each $n_{s}$. More concretely, assuming the scalar tilt parameter $n_{s}=0.963$ [57] and substituting into (57), for a particular choice of the constant $\kappa \sim 30$, we calculate two possible values of $\eta$ as follows:

(i) The first solution $\eta^{+}$has a positive value of $\sim 2.09 \times$ $10^{-2}$, which gives $\epsilon^{+} \sim 1.31 \times 10^{-2}$.

(ii) The second solution of $\eta^{-}$has a negative value of $\eta \sim$ $-9.34 \times 10^{-3}$, which leads to $\epsilon^{-} \sim 3.05 \times 10^{-3}$.

Surely both positive and negative values of $\eta$ give the same scalar tilt $n_{s} \sim 0.963$. Nevertheless, we can get two simultaneous tensor-to-scalar ratios: the first is $r^{+} \sim 0.21$, while the other is smaller $r^{-} \sim 4.9 \times 10^{-2}$. We conclude that the slow-roll inflationary models which are characterized by the proportionality $\epsilon \propto \eta^{2}$ can describe both the $E$-mode and the $B$-mode polarizations [79]; when a negative value of $\eta$ is observed near the peak of $\varphi$, it would need to be offset by a positive value of $\eta$ at some later time over a comparable field range in order to let $\epsilon$ be small again during the period of observable inflation. Generally, at low values of $\kappa$ the model predicts one small value of $r$ in addition to another higher value as required by the $B$-mode polarization inflationary models. Interestingly, at large values of $\kappa$ the model predicts a single value of the tensor-to-scalar parameter $r^{ \pm} \rightarrow 0.0987$, which agrees with the upper Planck limit $r_{0.002}<0.11$ at $95 \%$ CL.

Moreover, we can investigate the potential pattern which is characterized by the proportionality relation $\epsilon=\kappa \eta^{2}$. Recalling (55), this relation provides a simple differential equation with a solution

$V(\varphi)=A+B e^{ \pm 2 \sqrt{\frac{\pi}{\kappa}} \varphi}$, where $A$ and $B$ are constants of integration. In this way, we found that the Starobinsky model might be reconstructed naturally from observations if we want our model to describe $E$-mode and $B$-mode polarizations.

\subsection{The slow-roll parameters of the model}

We calculate the slow-roll parameters of the $V_{0}$-model to investigate its capability to predict a variable inflation. It can be shown that the $V_{0}$ potential (43) coincides with the potential constructed from Plank and BICEP2 observations (58). Using (43) and (55), we evaluate the slow-roll parameters

$$
\begin{aligned}
\epsilon_{0} & =\frac{24 \pi}{\left(12 \pi+\Lambda e^{-2 \sqrt{2 / 3}\left(\varphi-\varphi_{0}\right)}\right)^{2}}, \\
\eta_{0} & =\frac{4}{\left(12 \pi+\Lambda e^{-2 \sqrt{2 / 3}\left(\varphi-\varphi_{0}\right)}\right)} .
\end{aligned}
$$

From the slow-roll parameters (59) and (60) of the $V_{0}$-model, it can be shown that the model satisfies the proportionality $\epsilon=\kappa \eta^{2}$, where the proportionality constant $\kappa=\frac{3}{2} \pi$. This relation is not only independent of the values of $\Lambda$ but also it allows a vanishing cosmological constant to exist without affecting the generality of the proportionality relation. A similar relation has been obtained in the literature when studying the leading term behavior of the Starobinsky inflation as a special case of the $T$-models [80]. The number of $e$-folds from the end of inflation to the time of horizon crossing for observable scales is

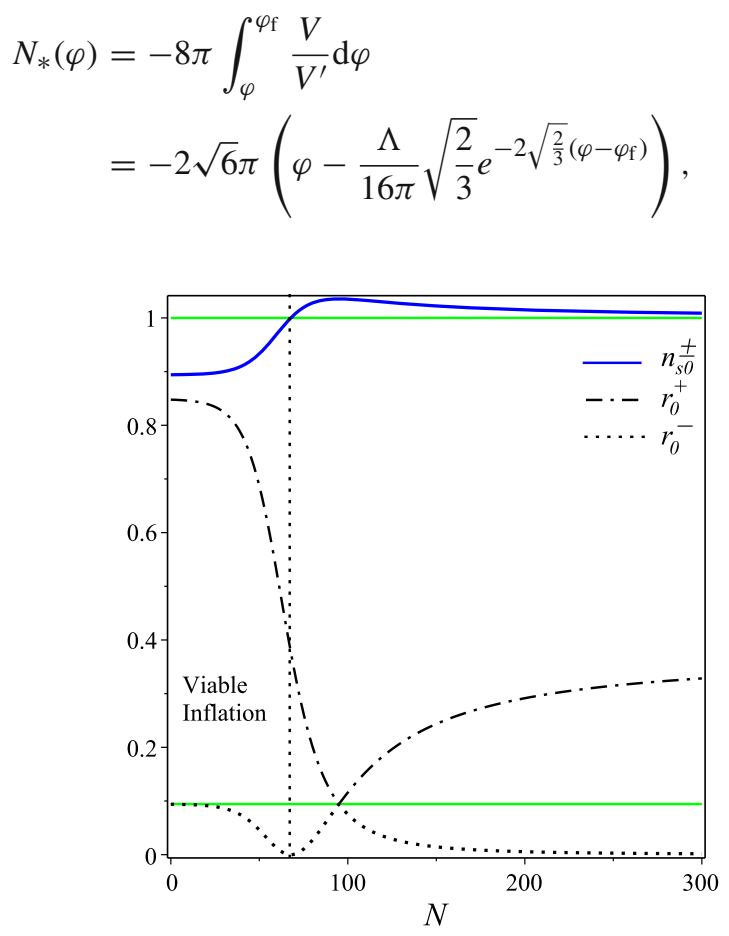

Fig. 3 The slow-roll parameters vs. the $e$-folding number of the $V_{0}$ model, where $\Lambda=10^{-30} \mathrm{~s}^{-2}$ 
Table 1 The predicted parameters of the $V_{0}$-model

\begin{tabular}{lllll}
\hline$N$ & 59 & 66 & 99 & $\infty$ \\
\hline$\eta_{0}^{+}$ & $8.48 \times 10^{-2}$ & $7.37 \times 10^{-2}$ & $3.85 \times 10^{-2}$ & $7.07 \times 10^{-2}$ \\
$\eta_{0}^{-}$ & $-1.40 \times 10^{-2}$ & $-3.00 \times 10^{-3}$ & $3.23 \times 10^{-2}$ & 0 \\
$\epsilon_{0}^{+}$ & $3.39 \times 10^{-2}$ & $2.56 \times 10^{-2}$ & $6.98 \times 10^{-3}$ & $2.36 \times 10^{-2}$ \\
$\epsilon_{0}^{-}$ & $9.27 \times 10^{-4}$ & $4.25 \times 10^{-5}$ & $4.90 \times 10^{-3}$ & 0 \\
$n_{s 0}^{ \pm}$ & 0.966 & 0.994 & 1.04 & $\preceq 1$ \\
$r_{0}^{+}$ & 0.542 & 0.410 & 0.112 & 0.377 \\
$r_{0}^{-}$ & 0.015 & $6.81 \times 10^{-4}$ & $7.85 \times 10^{-2}$ & 0 \\
\hline
\end{tabular}

where $\varphi_{\mathrm{f}}$ is the value of $\varphi$ at the end of inflation. Using (59)-(61) we can reexpress the slow-roll parameters as functions of $N$. At the end of inflation, i.e. $\operatorname{Max}(\epsilon,|\eta|)=1$, for the allowed range of $N_{*} \sim 60$ the observable cosmological wavelengths exit the Hubble radius for a minimum and maximum inflationary scale, and we have $\varphi_{\mathrm{f}}=40$. In the following steps we show the capability of the $\epsilon \propto \eta^{2} \bmod -$ els to predict two tensor-to-scalar ratios for a single spectral tilt at different $e$-folds. Using (61) we evaluate the spectral scalar tilt $n_{s 0}$ of the $V_{0}$-model as a function of $N$. Substituting $n_{s 0}(N)$ into (57) we evaluate two values $\eta_{0}^{+}$and $\eta_{0}^{-}$, for each we have two possible values $\epsilon_{0}^{+}$and $\epsilon_{0}^{-}$, respectively. Finally, we have two tensor-to-scalar ratios $r_{0}^{ \pm}=16 \epsilon_{0}^{ \pm}$, while the two expected spectral scalar tilts are identical, i.e. $n_{s 0}^{+}=n_{s 0}^{-}$. This procedure enables one to draw the plots of Fig. 3 . The plots represent the evolution of the observable parameters vs. the $e$-folding number. It is clear that the spectral scalar tilt has a single pattern represented by the $n_{s 0}^{ \pm}$plot, while the tensorto-scalar ratio has two distinguished patterns represented by the $r_{0}^{+}$and $r_{0}^{-}$plots. The $r_{0}^{+}$has a lower limit $>0.094$, which is in agreement with the $B$-mode polarization models. On the other hand, the $r_{0}^{-}$plot has an upper limit $<0.094$, which is in agreement with the $E$-mode polarization models.

In Fig. 3, the viable inflation is characterized by $n_{s 0}^{ \pm}<1$ region. The region with $n_{s 0}^{ \pm}$exceeding unity is $\operatorname{Max}\left(n_{s 0}^{ \pm}\right)<$ 1.04; although this small deviation from the scale invariance of the scalar power spectrum is acceptable at the level of theoretical predictions, it can be excluded as it appears to be beyond the acceptable range of the number of $e$-folds $N>$ 60 . Interestingly, at large $e$-folding number the spectral scalar tilt goes again below unity, so that $\left(1-n_{s 0}^{ \pm}\right) \sim 4 \times 10^{-10}$. We highlight some results in Table 1 showing that the $V_{0^{-}}$ model can predict large tensor-to-scalar ratios of the $B$-mode polarization as well as small ratios as required by the $E$-mode polarization.

A similar procedure can be applied to the $V_{1}$-model where the $\epsilon=\kappa \eta^{2}$ relation is still valid with larger proportionality constant $\kappa=6 \pi$. Accordingly, for the tensor-to-scalar ratios that can be achieved, e.g. at $N=55$, we have $\left(r_{1}^{-}, r_{1}^{+}, n_{s 1}^{ \pm}\right)$ $=\left(3.23 \times 10^{-2}, 0.237,0.967\right)$ where the scalar tilt in this case always has $n_{s 1}^{ \pm}<1$. Also, this procedure can be used to put constraints on the choice of the conformal weight in the power law and Starobinsky inflation models from the CMB observations ${ }^{4}$.

\section{Discussions and final remarks}

We investigate a special class of $f(T)$ gravity governed by FLU assumptions. In this model, we assume the vanishing of the coefficient of the sectional curvature in the modified Friedmann equations. This enables one to identify a particular class of the $f_{n}(T) \propto \sum \frac{1}{\sqrt{-T^{n}}}$ gravity, usually hidden by the SFU assumption, cannot be covered by TEGR theory. The FLU model, at large Hubble regime, is consistent with the cosmic inflation scenario. Also, it enables one to evaluate the dynamical EoS of the cosmic fluid. In the particular case $n=3$, the fluid can evolve from a phantom initial phase crossing the phantom divide line $(\omega=-1)$ to radiation and possibly stiff matter with a quintessence fate. As a matter of fact, the dynamical EoS avoids the usual problems of the cosmological constant DE models. In addition, there is no need to assume a large cosmological constant at early times for the universe.

We provide an alternative approach to study $f(T)$ inflation by considering the case when the torsion consists of a scalar field $\varphi$. This approach allows the $f(T)$ to predict the inflationary observable parameters. We show that the teleparallel torsion scalar can couple to the kinetic energy of the scalar field, while its potential $V(\varphi)$ can be induced by the $f(T)$ gravity. In this case, we define a scalar field sensitive to the spacetime symmetry with a potential induced exactly from extended teleparallel gravity. This leads finally to a gravitational quintessence model governed by the Friedmann and Klein-Gordon equations.

The quintessence model obtained covers different inflationary models according to the expansion limit. In this study, we give more attention to the $V_{0}$-model. It has been shown that the $V_{0}$ model relates the slow-roll parameters by the proportionality $\epsilon=\kappa \eta^{2}$, which is in agreement with the model

\footnotetext{
${ }^{4}$ This work is in progress now.
} 
constructed from Planck and BICEP2 data. The model can be classified as power law inflation, which has been shown to be capable of performing two tensor-to-scalar ratios consistent with both the $E$ and $B$ modes of polarization, while it predicted a scalar spectral index that is still unique. In particular, the observable parameters evolution with the $e$-folding number $N$ is shown in Fig. 3 and Table 1. We just highlighted some results of the other models, while further studies are left for future work. Higher-order potentials interpolate between Starobinsky and quadratic-like inflations.

Finally, we would like to mention that at large $\kappa$ the model predicts a single tensor-to-scalar parameter $r \rightarrow 0.0987$, in agreement with the Planck observation limit. This result can be used to constrain the choice of the conformal weight in power law and Starobinsky models to the observable parameters of the cosmic inflation epoch.

Acknowledgments The authors would like to thank the anonymous referee for her/his valuable comments, which, indeed, helped to improve the work. This article is partially supported by the Egyptian Ministry of Scientific Research under project No. 24-2-12.

Open Access This article is distributed under the terms of the Creative Commons Attribution 4.0 International License (http://creativecomm ons.org/licenses/by/4.0/), which permits unrestricted use, distribution, and reproduction in any medium, provided you give appropriate credit to the original author(s) and the source, provide a link to the Creative Commons license, and indicate if changes were made. Funded by SCOAP ${ }^{3}$.

\section{References}

1. A.G. Riess, A.V. Filippenko, P. Challis et al., Astrophys. J. 116, 1009 (1998). arXiv:astro-ph/9805201

2. R. Aldrovandi, J.G. Pereira, in Teleparallel Gravity: An Introduction. Fundamental Theories of Physics, vol 173 (Springer, Dordrecht, 2013)

3. B. Li, T.P. Sotiriou, J.D. Barrow, Phys. Rev. D 83(6), 064035 (2011). arXiv:1010.1041

4. T.P. Sotiriou, B. Li, J.D. Barrow, Phys. Rev. D 83(10), 104030 (2011). arXiv:1012.4039

5. A. Einstein, Riemann-Geometrie mit Aufrechterhaltung des Begriffes des Fernparallelismus (Phys.-math, Klasse Preussische Akademie der Wissenschaften, Sitzungsberichte, 1928), pp. 217221

6. F.I. Mikhail, Relativistic cosmology and some related problems in general relativity. Ph. D. Thesis, University of London (1952)

7. W.H. McCrea, F.I. Mikhail, R. Soc. Lond. Proc. Ser. A 235, 11 (1956)

8. F. Mikhail, Al Nuovo Cimento Ser. X 32, 886 (1964)

9. F.I. Mikhail, M.I. Wanas, R. Soc. Lond. Proc. Ser. A 356, 471 (1977)

10. C. Møller, K. Dan, Vidensk. Selsk. Mat. Fys. Skr. 89, 13 (1978)

11. K. Hayashi, T. Shirafuji, Phys. Rev. D 19, 3524 (1979)

12. R. Utiyama, Phys. Rev. 101(5), 1597 (1956)

13. T.W.B. Kibble, J. Math. Phys. 2(2), 212 (1961)

14. D.W. Sciama, Rev. Mod. Phys. 463-469 (1964)

15. F.W. Hehl, P. von der Heyde, G.D. Kerlick et al., Rev. Modern Phys. 48, 393 (1976)

16. R. Utiyama, Progress Theor. Phys. 64, 2207 (1980)
17. N.L. Youssef, A.M. Sid-Ahmed, Rep. Math. Phys. 60, 39 (2007). arXiv:gr-qc/0604111

18. N.L. Youssef, W.A. Elsayed, Rep. Math. Phys. 72, 1 (2013). arXiv:1209.1379

19. M.I. Wanas, Modern Phys. Lett. A 24, 1749 (2009). arXiv:0801.1132

20. M.I. Wanas, M.M. Kamal, Modern Phys. Lett. A 26, 2065 (2011). arXiv: 1103.4121

21. N.L. Youssef, A.M. Sid-Ahmed, E.H. Taha, Int. J. Geom. Meth Mod. Phys. 10(7), 1350029 (2013). arXiv:1206.4505

22. R. Ferraro, F. Fiorini, Phys. Rev. D 75(8), 084031 (2007). arXiv:gr-qc/0610067

23. R. Ferraro, F. Fiorini, Phys. Rev. D 78(12), 124019 (2008). arXiv:0812.1981 [gr-qc]

24. G.R. Bengochea, R. Ferraro, Phys. Rev. D 79(12), 124019 (2009). arXiv:0812.1205

25. E.V. Linder, Phys. Rev. D 81(12), 127301 (2010). arXiv:1005.3039

26. K. Bamba, C.-Q. Geng, C.-C. Lee, ArXiv e-prints (2010). arXiv: 1008.4036

27. K. Bamba, C.-Q. Geng, C.-C. Lee et al., JCAP 1, 021 (2011). arXiv: 1011.0508

28. R.-J. Yang, EPL (Europhys. Lett.) 93, 60001 (2011). arXiv: 1010.1376

29. S.-H. Chen, J.B. Dent, S. Dutta, et al., Phys. Rev. D 83(2), 023508 (2011). arXiv:1008.1250

30. R. Ferraro, F. Fiorini, Phys. Rev. D 84(8), 083518 (2011). arXiv: 1109.4209

31. R. Ferraro, F. Fiorini, Phys. Lett. B 702, 75 (2011). arXiv: 1103.0824

32. L. Iorio, E.N. Saridakis, Mon. Not. R. Astron. Soc. 427, 1555 (2012). arXiv:1203.5781

33. S. Capozziello, P.A. González, E.N. Saridakis et al., JHEP 2, 39 (2013). arXiv: 1210.1098

34. G.G.L. Nashed, Phys. Rev. D 88(10), 104034 (2013). arXiv: 1311.3131

35. G.G.L. Nashed, Gen. Relat. Gravit. 45, 1887 (2013) arXiv: 1502.05219

36. M.E. Rodrigues, M.J.S. Houndjo, J. Tossa et al., JCAP 11, 024 (2013). arXiv:1306.2280

37. G.G.L. Nashed, EPL (Europhys. Lett.) 105, 10001 (2014). arXiv: 1501.00974

38. C. Bejarano, R. Ferraro, M.J. Guzmán, Eur. Phys. J. C 75, 77 (2015). arXiv: 1412.0641

39. G.G.L. Nashed, J. Phys. Soc. Jpn. 84(4), 044006 (2015)

40. G.G.L. Nashed, Int. J. Modern Phys. D 24, 1550007 (2015)

41. K. Bamba, S. Capozziello, S. Nojiri et al., Astrophys. Space Sci. 342, 155 (2012). arXiv:1205.3421

42. K. Bamba, S. Nojiri, S.D. Odintsov, Phys. Lett. B 731, 257-264 (2014). arXiv: 1401.7378

43. K. Bamba, S.D. Odintsov (2014). arXiv:1402.7114

44. M. Jamil, D. Momeni, R. Myrzakulov, Int. J. Theor. Phys. (2014). arXiv: 1309.3269

45. T. Harko, F.S.N. Lobo, G. Otalora, et al., Phys. Rev. D 89(12), 124036 (2014). arXiv: 1404.6212

46. G.G.L. Nashed, W. El Hanafy, Eur. Phys. J. C 74(10), 3099 (2014). arXiv: 1403.0913

47. M.I. Wanas, H.A. Hassan, Int. J. Theor. Phys. 53, 3901 (2014)

48. W. El Hanafy, G.G.L. Nashed (2014). arXiv:1410.2467

49. Y. Wu, Z.-C. Chen, J. Wang et al. (2015). arXiv:1503.05281

50. E.L.B. Junior, M.E. Rodrigues, M.J.S. Houndjo (2015). arXiv:1503.07427

51. Y.-F. Cai, S.-H. Chen, J.B. Dent et al., Class. Quantum Gravity 28(21), 215011 (2011). arXiv:1104.4349

52. Y.-F. Cai, J. Quintin, E.N. Saridakis et al., JCAP 7, 033 (2014). arXiv: 1404.4364

53. G. Nashed, W. El Hanafy, S. Ibrahim (2014). arXiv:1411.3293 
54. G. Kofinas, E.N. Saridakis, Phys. Rev. D 90(8), 084044 (2014). arXiv:1404.2249

55. G. Kofinas, E.N. Saridakis, Phys. Rev. D 90(8), 084045 (2014). arXiv:1408.0107

56. G. Kofinas, G. Leon, E.N. Saridakis, Class. Quantum Gravity 31(17), 175011 (2014). arXiv: 1404.7100

57. Planck Collaboration, P.A.R. Ade, N. Aghanim et al., Astron. Astrophys. 571, A22 (2014). arXiv:1303.5082

58. Planck Collaboration, P.A.R. Ade, N. Aghanim et al. Astron. Astrophys. 571, A16 (2014). arXiv: 1303.5076

59. BICEP2 Collaboration, P.A.R. Ade et al., Phys. Rev. Lett. 112(24), 241101 (2014). arXiv:1403.3985

60. J.W. Maluf, Annalen der Physik 525, 339 (2013). arXiv:1303.3897

61. H.P. Robertson, Ann. Math. 33, 496 (1932)

62. O. Farooq, D. Mania, B. Ratra, Astrophys. Space Sci. 357, 11 (2015)

63. J.N. Dossett, M. Ishak, Phys. Rev. D 86(10), 103008 (2012). arXiv:1205.2422

64. J.-F. Zhang, M.-M. Zhao, J.-L. Cui et al., Eur. Phys. J. C 74, 3178 (2014). arXiv:1409.6078

65. V. Sahni, Class. Quantum Gravity 19, 3435 (2002). arXiv:astro-ph/0202076

66. M.I. Wanas, Adv. High Energy Phys. 2012, Article ID 752613 (2012)

67. H.-J. Xie, T. Shirafuji (1996). arXiv:gr-qc/9603006

68. V.E. Rochev, Theor. Math. Phys. 18, 160(1974)
69. S. Hojman, M. Rosenbaum, M.P. Ryan et al., Phys. Rev. D 17, 3141 (1978)

70. R.T. Hammond, Class. Quantum Gravity 7, 2107 (1990)

71. F.W. Hehl, Y.N. Obukhov, in Gyros, Clocks, Interferometers ...: Testing Relativistic Gravity in Space ed. by C. Lämmerzahl, C.W.F. Everitt, F.W. Hehl, vol 562. Lecture Notes in Physics (Springer, Berlin, 2001), p. 479. arXiv:gr-qc/0001010

72. V.C. de Andrade, J.G. Pereira, Gen. Relat. Gravit. 30, 263 (1998). arXiv:gr-qc/9706070

73. A. Einstein, The Meaning of Relativity, 5th edn (The Princeton University Press, Princeton, 1955)

74. J.B. Fonseca-Neto, C. Romero, S.P.G. Martinez, Gen. Relat. Gravit. 45, 1579 (2013). arXiv:1211.1557

75. L.Y. Nabil, A.M. Sid-Ahmed, in The International Conference on Developing and Extending Einstein's Ideas. Egyptian Relativity Group (NART, ed. by M. Wanas (Cairo, Egypt, 2005), pp. 19-21

76. R. Kallosh, A. Linde, D. Roest, JHEP 8, 52 (2014). arXiv: 1405.3646

77. A.A. Starobinsky, Phys. Lett. B 91, 99 (1980)

78. I. Zlatev, L. Wang, P.J. Steinhardt, Phys. Rev. Lett. 82, 896 (1999). arXiv:astro-ph/9807002

79. R. Bousso, D. Harlow, L. Senatore, Phys. Rev. D 91(8), 083527 (2015). arXiv:1309.4060

80. Y.-F. Cai, J.-O. Gong, S. Pi, Phys. Lett. B 738, 20 (2014). arXiv: 1404.2560 